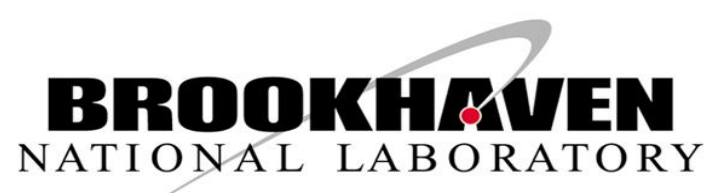

BNL-113491-2017-CP

\title{
Software Tools for X-ray Photon Correlation and X-ray Speckle Visibility Spectroscopy
}

Sameera K. Abeykoon, Yugang Zhang, Eric D. Dill, Thomas A. Caswell, Daniel B. Allan, Arman Akilic, Lutz Wiegart, Stuart Wilkins, Annie Heroux, Kerstin K. van Dam, Mark Sutton and Andrei Fluerasu

Presented at the New York Scientific Data Summit 2016 (NYSDS2016)

New York, NY

August 14-17, 2016

August 2016

Computational Science Initiative

Brookhaven National Laboratory

\author{
U.S. Department of Energy \\ USDOE Office of Science, \\ Advanced Scientific Computing Research
}

Notice: This manuscript has been authored by employees of Brookhaven Science Associates, LLC under Contract No. DE- SC0012704 with the U.S. Department of Energy. The publisher by accepting the manuscript for publication acknowledges that the United States Government retains a non-exclusive, paid-up, irrevocable, world-wide license to publish or reproduce the published form of this manuscript, or allow others to do so, for United States Government purposes. 


\section{DISCLAIMER}

This report was prepared as an account of work sponsored by an agency of the United States Government. Neither the United States Government nor any agency thereof, nor any of their employees, nor any of their contractors, subcontractors, or their employees, makes any warranty, express or implied, or assumes any legal liability or responsibility for the accuracy, completeness, or any third party's use or the results of such use of any information, apparatus, product, or process disclosed, or represents that its use would not infringe privately owned rights. Reference herein to any specific commercial product, process, or service by trade name, trademark, manufacturer, or otherwise, does not necessarily constitute or imply its endorsement, recommendation, or favoring by the United States Government or any agency thereof or its contractors or subcontractors. The views and opinions of authors expressed herein do not necessarily state or reflect those of the United States Government or any agency thereof. 


\title{
Software Tools for X-ray Photon Correlation and X-ray Speckle Visibility Spectroscopy
}

\author{
Sameera K. Abeykoon, Yugang Zhang, Eric D. Dill, Thomas A. Caswell, Daniel B. Allan, Arman Akilic, \\ Lutz Wiegart, Stuart Wilkins, Annie Heroux, Kerstin K. van Dam, Mark Sutton and Andrei Fluerasu
}

\begin{abstract}
A set of new data analysis software tools have been developed for the study of structural dynamics of materials using coherent scattering and photon correlation techniques. The new software tools can readily process high-throughput, multidimensional data, enabling studies of slow and fast dynamics of materials using X-ray Speckle Visibility Spectroscopy and Xray Photon Correlation Spectroscopy techniques. They support a wide range of user expertise, from novice to developer, and are available in Scikit-beam python package which is available at https://github.com/scikit-beam/scikit-beam.
\end{abstract}

Index Terms-Scikit-beam, X-ray Speckle Visibility Spectroscopy, X-ray Photon Correlation Spectroscopy

\section{INTRODUCTION}

For a long time it has been widely appreciated that the macroscopic properties of materials are directly related to their nano-scale and micro-scale structure and dynamics. While "traditional" X-ray scattering experiments have been used for over a century, it is only with the advent of third generation synchrotron sources that the beam brightness enabled coherent $\mathrm{X}$-ray scattering experiments. This opens the possibility to measure not only time-averaged structural information but also time-scales associated with nanoscale and meso-scale fluctuations (dynamics) [1]. These new type of studies and other experiments enabled by the ultra-high brightness synchrotron sources such as the National Synchrotron Light Source-II (NSLS-II) at Brookhaven National Laboratory (BNL) bring a whole series of new challenges associated with the data management and experimental data analysis [2]. For each particular experiment the data rates typically reach tens to hundreds of gigabytes/hour and several terabytes/day, which is relatively modest compared to, say, high energy particle physics experiments. However, the challenge comes with the fact that a synchrotron such as NSLS-II will operate at its full capacity with 50-60 beamlines working in parallel. Each beamline is typically run by a relatively small team of scientists focusing on different experiments with completely different scientific objectives but which can all benefit from a common "basic experimental toolkit" for data management and data analysis. To address this challenge, the NSLS-II is developing

S. K. Abeykoon (e-mail: sameera@bnl.gov) and K. K. van Dam are with the Computer Science Initiative, Brookhaven National Laboratory, Upton, NY, 11973, USA.

Y. Zhang, E. D. Dill, T. A. Caswell, D. B. Allan, A. Akilic, L. Wiegart, S. Wilkins, A. Heroux and A. Fluerasu (e-mail: fluerasu@bnl.gov) are with the Photon Sciences Department, Brookhaven National Laboratory, Upton, NY, 11973, USA.

M. Sutton is with the Physics Department, McGill University, Montreal, Canada H3A 2T8 a high performance data management structure capable of handling, processing, visualization, analysis, and storage of high-throughput data to optimize the scientific productivity of its beamlines. The Scikit-beam is a package developed by this effort, and the data analysis tools described here focus on X-ray Speckle Visibility Spectroscopy (XSVS) and X-ray Photon Correlation Spectroscopy (XPCS) for coherent X-ray scattering.

The basic idea behind these techniques is that coherent light scattered by a heterogeneous sample produces a speckle pattern characteristic for the specific arrangement of the scatterers. As the scatterers fluctuate over time, the resultant speckle intensity will change and time autocorrelation functions of the speckle fluctuations will reveal the wave vector-dependent timescales associated with the motion of the scatterers. This is the basis of the XPCS technique. If the speed of the area detector used to measure the speckle patterns cannot sustain the time rates associated with the speckle fluctuations, an alternative technique - XSVS - can be used. Here the contrast of single speckle patterns measured with different integration times are compared with each other. When motions in the sample occur on timescales comparable with the integration times the speckles will appear "blurred" and the speckle contrast will be reduced, revealing the time-scales associated with these motions.

Both the XPCS and XSVS methods are "photon starved" techniques and benefit directly from the high brightness and enhanced coherent flux of the NSLS-II source. In the photon energy range where the Coherent Hard X-ray (CHX) beamline operates, $6-16 \mathrm{keV} \mathrm{[3]} \mathrm{the} \mathrm{source} \mathrm{brightness} \mathrm{exceeds} \mathrm{by} \mathrm{more}$ than one order of magnitude that of other facilities such as the Advanced Photon Source(APS) at Argonne National Laboratory or the European Synchrotron Radiation Facility(ESRF) in Grenoble, France and, as a result, the timescales accessible from a coherent scattering experiment can be extended by more than two orders of magnitude compared to similar experiments performed at those facilities ([3], [4]).

Large-scale data management, analysis, and computation is increasingly important in scientific research. This is driven by both theory and experiment. Theory driven material discovery [5] and the Materials Genome Initiative ([6], [7]) can generate large amounts of simulated and in situ scattering data on predicted materials. In parallel improvements in instrumentation and novel experimental techniques, such as XSVS and XPCS, will generate large volumes of experimental data. These applications will require processing, analyzing, and interpreting a continuous stream of structured and unstructured 
data.

\section{A. Coherent Hard X-ray Beamline}

The XPCS and XSVS experiments were performed at the 11-ID, Coherent Hard X-ray (CHX) beamline of the NSLSII [3]. The X-ray source at the CHX beamline is a $3 \mathrm{~m}$ long in-vacuum undulator with $20 \mathrm{~mm}$ magnetic period. A double crystal $S i(111)$ monochromator was used to tune the $\mathrm{X}$-ray energy to $9 \mathrm{KeV}$ with a relative bandwith of $\Delta \lambda / \lambda$ $=10^{-4}$ and a flux of $\simeq 10^{11}$ photons per second for $\mathrm{a}$ storage ring current of $250 \mathrm{~mA}$. A transversely partial coherent beam was tailored to a spot size of $15 \mu \mathrm{m} \times 15 \mu \mathrm{m}$ using a combination of beryllium compound refractive lenses for vertical focusing, $S i$ kinoform lenses for horizontal focusing and a series of beam-defining and beam-collimating slits. The scattered X-rays were recorded by a photon-counting Dectris Eiger 4M area detector with 75 um square pixel located $4.8 \mathrm{~m}$ downstream of the sample. The detector can sustain a fullframe and full dynamic range (12 bit) detection rate of $750 \mathrm{~Hz}$ resulting in an uncompressed data rate of $4.5 \mathrm{~GB} / \mathrm{s}$.

The first sample used here was a thin piece of SCHOTT CoralPor $\AA$ porous glass [8] with an average pore size of about $31 \mathrm{~nm}$. Fig. 1 shows a speckle pattern from the CoralPor $\mathbb{R}$ sample. The large ring-shaped scattering measures a correlation length associated with the average pore size while the speckles are associated with the exact distribution of pores. As this sample is expected to be static, it was used as a reference and for the measurement of the speckle contrast.

The XPCS studies were done with a sample prepared from a suspension of charge stabilized colloidal silica spheres with a radius of $250 \mathrm{~nm}$. The colloids were purchased from Duke Scientific in an aqueous low-concentration solution, were concentrated by centrifugation, and redispersed in a mixture solution of water and glycerol with weight ratio about 1:9. The final concentration of the sample was kept low $(\sim 1 \%$ volume fraction), which resulted in the colloids undergoing a well understood Brownian diffusive motion. The suspensions were filled in quartz capillaries and placed in the X-ray beam. The scattering from this colloids is shown in Fig. 2. As the sample is isotropic, its structural properties are well described by a circular average over the scattered intensity.

\section{THEORY}

\section{A. X-ray Photon Correlation Spectroscopy}

XPCS has emerged as a powerful experimental tool for the study of nano- and meso-scale dynamics in materials. Compared to other techniques that can be used to measure structural dynamics, XPCS can be used to access a unique combination of length and timescales which are difficult or impossible to measure otherwise ( [9], [1].) This technique can be used to study both equilibrium and non-equilibrium dynamics [10].

1) One-time Auto Correlation Function: In XPCS, the sample dynamics is quantified by the normalized one-time correlation function defined as [4],

$$
g^{(2)}(q, t)=\frac{\left\langle I\left(q, t_{0}\right) I\left(q, t_{0}+t\right)\right\rangle}{\left\langle I\left(q, t_{0}\right)\right\rangle^{2}} .
$$

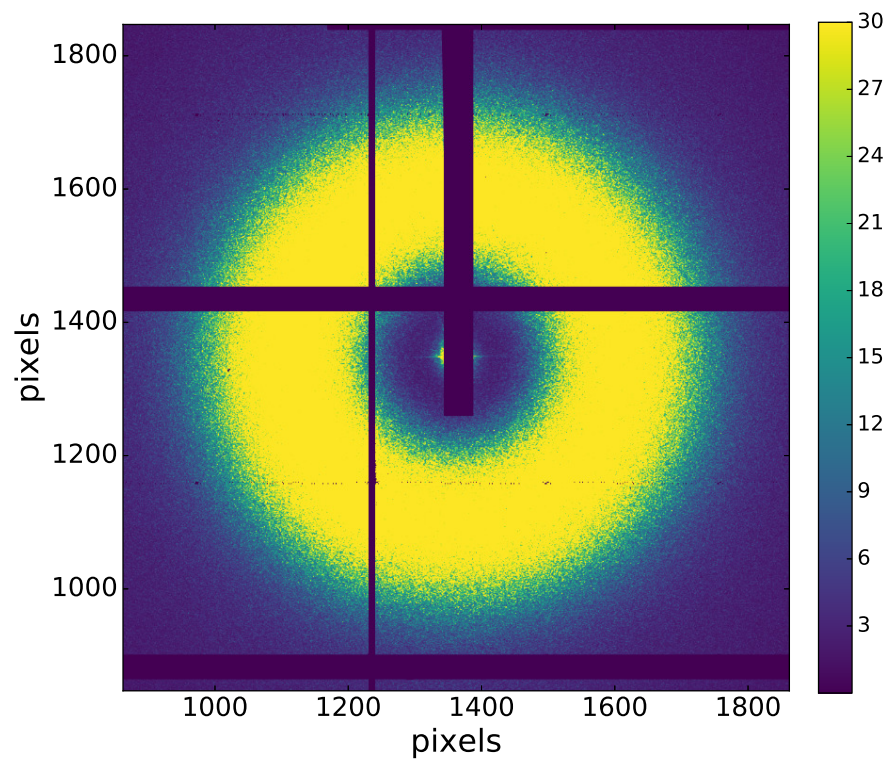

Fig. 1. A Speckle Pattern (averaged over 10000 images with

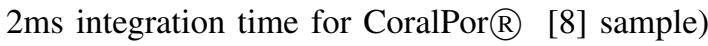

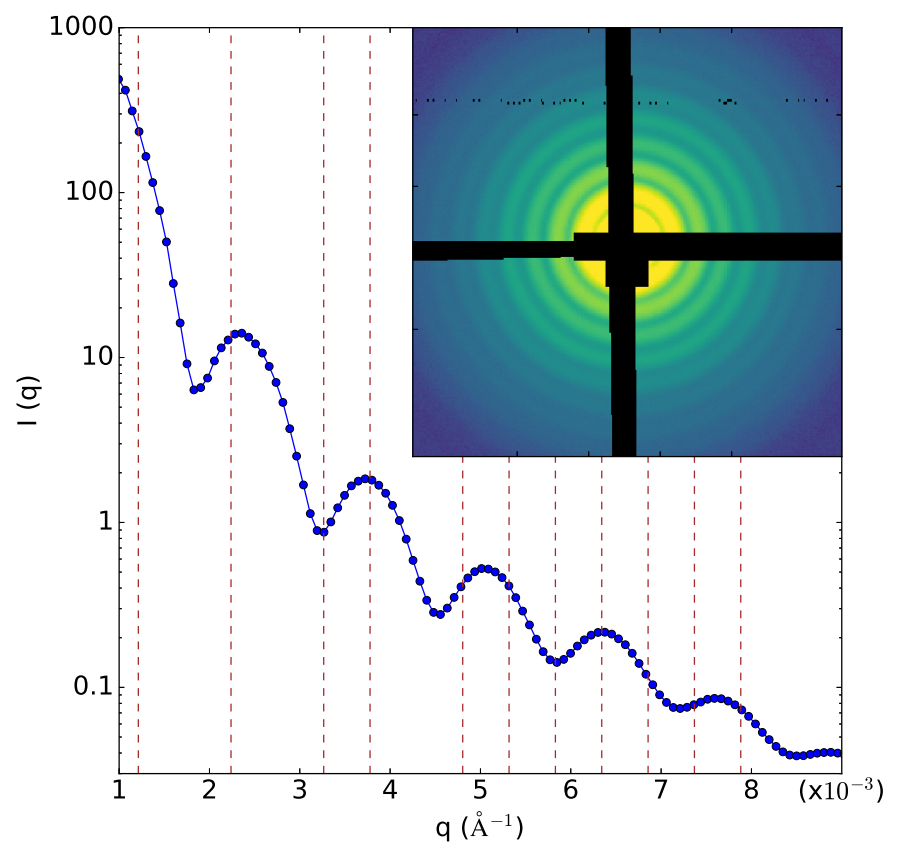

Fig. 2. Scattered $I(q)$ from the Silica colloidal suspension calculated by performing a radial average over the timeaveraged 2D scattering pattern (inset). The dashed vetical lines show the 11 values of the momentum transfer (" $q$-rings") which were used in the XPCS analysis.

Here \langle\rangle denotes an ensemble average over the "initial" time $t_{0}$ and over nominally equivalent pixels; $I\left(q, t_{0}\right)$ is the scattered intensity at momentum transfer $q$ and time $t_{0}$.

Theoretical simulations typically calculate the field one-time correlation function, also known as intermediate scattering function (ISF) or dynamic structure factor $g^{(1)}(q, t)$ which is related (assuming Gaussian statistics for the intensity fluctua- 
tions) to the intensity autocorrelation function by the Siegert relation ( [11]),

$$
g^{(2)}(q, t)=\beta_{2}\left[g^{(1)}(q, t)\right]^{2}+g_{\infty}
$$

where $\beta_{2}$ is the correlation function contrast factor, and $g_{\infty}$ is the baseline which is equal to 1 for ergodic samples [12].

As the ISF describes all the dynamical information about a system and hence one can say that the XPCS method provides a direct measurement of the nano- and micro- scale dynamics of the scatterers.

In many cases, when the scatterers are undergoing a simple free-diffusive (a.k.a. Brownian) motion, the ISF can be described by a simple exponential decay [12]

$$
g^{(1)}(q, t)=\exp [-\gamma(q) t]
$$

where $\gamma(q)$ is a relaxation rate related to the diffusion coefficient $D_{0}$ and the momentum transfer $q$ by

$$
\gamma(q)=D_{0} q^{2}
$$

By combining equations 2,3 , and 4 , the intensity autocorrelation function can be written as,

$$
g^{(2)}(q, t)=\beta_{2} \exp \left[-2 D_{0} q^{2} t\right]+g_{\infty} .
$$

The calculated correlation functions and fits with equation 5 are shown in Figure 3.

2) Two-time Correlation Function: In out-of-equilibrium systems, where the one-time correlation functions change with time, the dynamics can be characterized by two-time correlation functions defined as ( [10], [13]),

$$
C\left(q, t_{1}, t_{2}\right)=\frac{\left\langle I\left(q, t_{1}\right) I\left(q, t_{2}\right)\right\rangle}{\left\langle I\left(q, t_{1}\right)\right\rangle\left\langle I\left(q, t_{2}\right)\right\rangle} .
$$

Here $<>$ denotes an ensemble average over equivalent pixels of the detector, (e.g. all having, within a small variation, the same $q$ ). In many situations, it is advantageous to think about a two-time correlation function in terms of two re-scaled time variables. An average time or "age" of the sample can be defined as $t_{a}=\left(t_{1}+t_{2}\right) / 2$ and represents the time along the $t_{1}=t_{2}$ diagonal on a two-time correlation as shown in Fig. 4. The role of the time variable $t$ in $g^{(2)}(q, t)$ is now taken by the time difference $t=\left|t_{1}-t_{2}\right|$, which measures the "distance" from the $t_{1}=t_{2}$ diagonal. When the system is in equilibrium, the two-time correlation functions depend only on the time difference $t$, and not on the sample "age" $t_{a}$ [10]. In this case, the one-time correlation function $g^{(2)}(q, t)$ is simply an average of $C\left(q, t_{1}, t_{2}\right)$ over all ages $t_{a}$ ( [10], [13]).

\section{B. X-ray Speckle Visibility Spectroscopy}

While XPCS is the most common technique used to study the dynamics of materials, the method has certain limitations. As explained in the previous section, XPCS requires a long series of images (i.e. a movie) which are used to calculate the intensity fluctuations autocorrelation functions. As a consequence, the minimum correlation time accessible in XPCS is limited by the maximum frame rate of the area detector.
In laser light scattering, speckle visibly spectroscopy (SVS) was proposed as an experimental technique allowing to quantify the sample dynamics from a series of single scattering patterns [17]. Following this development, X-ray speckle visibility spectroscopy (XSVS) has been recently introduced also to coherent X-ray scattering( [14], [15], [16], [12]).

The main idea behind XSVS is that dynamical information is "encoded" even in single speckle patterns. Indeed, as an image is recorded by the detector using an integration time, or exposure time of $T$, the speckle contrast, or "speckle visibility" will appear "blurred" if there is motion in the sample occurring on the same timescales. A speckle visibility experiment can then be performed by repeating the single speckle detection process with different integration times and following the decay of the speckle contrast with $T$. As for most photon counting area detectors the shortest integration (exposure) time can be orders of magnitude faster than the period of a continuous sequence of images, XSVS can be used to push the capabilities of a coherent scattering experiment towards faster timescales than XPCS.

The speckle contrast seems to be readily available by simply calculating the variance of intensity distribution over a region of equivalent pixels. However, this quantity is affected by experimental noise and is not an accurate measurement of the speckle visibility. The effect of the noise is stronger when the counts are low which is, unfortunately, often the case with XSVS experiments. To correctly quantify the speckle contrast one then has to take into account the statistical nature of the photon detection process. Essentially, the probability of measuring a number of counts $A$ in a given region of the detector is a convolution between the Poisson distribution which describes the experimental noise and the so-called Gamma distribution, which describes the intensity variation associated with partially coherent interference (speckle). The details of the XSVS methods where described by $\mathrm{Li}$ et. al. [12] following the SVS formalism for laser light scattering introduced by Bandyopadhyay et. al. [17] and earlier work by Mandel [18], [19].

For the purpose of the work described here it is sufficient to note that the intensity distribution in the X-ray speckle patterns recorded in XSVS is described by the Poisson-Gamma, also known as the negative-binomial distribution function [19] which is given by (see Fig. 5)

$$
P(A)=\frac{\Gamma(A+M)}{\Gamma(A+1) \Gamma(M)}\left(\frac{M}{\langle A\rangle+M}\right)^{M}\left(\frac{\langle A\rangle}{M+\langle A\rangle}\right)^{A} .
$$

Here $P(A)$ is the probability density of detecting $A$ photon events in a given region of the detector (typically a pixel at momentum transfer $q$ ), for a certain integration time, $T, \Gamma$ is the gamma function, $\langle A\rangle$ is the average number of photon counts and $M$ is typically referred to as the "number of coherent modes", which is related to the $T$-dependent and $q$ dependent contrast factor by

$$
\beta(q, T)=\frac{1}{M} .
$$

In a specific experiment, the contrast factor $\beta$ is extracted by fitting the experimental probability density with equation 7 

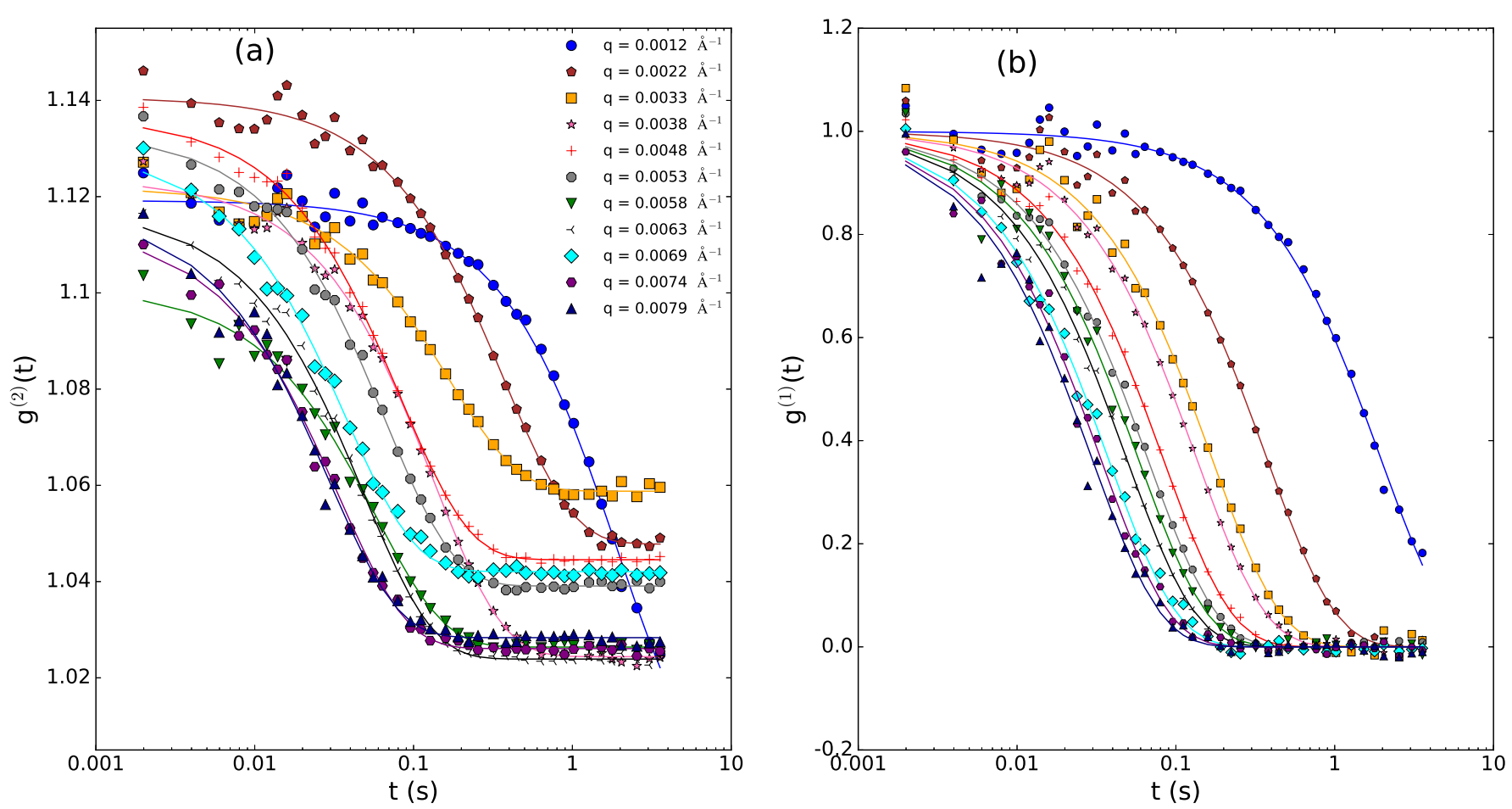

Fig. 3. (a)Multi-tau one-time correlation functions, $g^{(2)}(q, t)$ 's calculated using the Scikit-beam XPCS analysis tools and plotted as a function of time delay, $t$. The 11 correlation functions shown here correspond to $q$ rings with width of $\Delta q \simeq 1.0 \times 10^{-4} \AA^{-1}$. Solid lines represent the fitted curves using equation 5. (b) ISFs $g^{(1)}(q, t)$ (equation 12) for the same $11 q$ values.

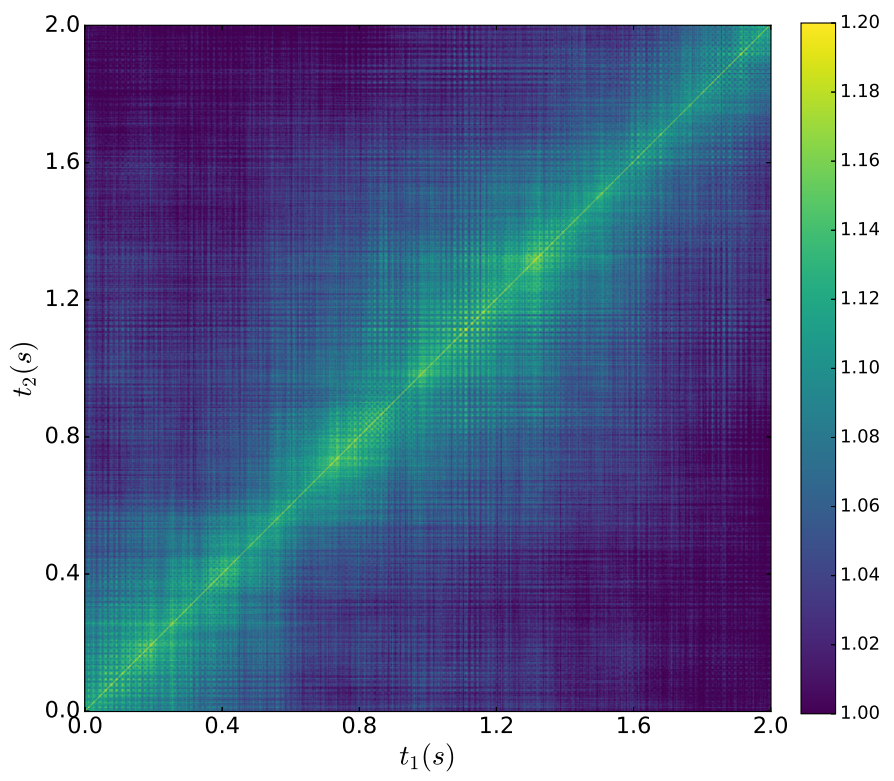

Fig. 4. Two-time correlation function $C\left(\mathrm{q}=4.80 \times 10^{-3} \AA^{-1}\right.$, $\left.t_{1}, t_{2}\right)$ for calculated from the silica colloid data.

for speckle images recorded with different integration times $T$. According to Mandel [18] and Bandyopadhyay et. al. [17], and assuming Gaussian statistics, the contrast factor can be written as a function of ISF,

$$
\beta(q, T)=\beta_{1} \int_{0}^{T} 2(1-t / T)\left[g^{(1)}(q, t)\right]^{2} d t / T+\beta_{\infty} .
$$

Here $\beta_{1}$ is the $T \rightarrow 0$ limit of the speckle contrast factor and $\beta_{\infty}$ is the baseline and is equal to zero when the sample is ergodic and does not generate a static, "frozen-in", speckle pattern.

Equation 9 leads to various analytic expressions [17] corresponding to the exact nature of the dynamical processes that can take place. When samples undergo a simple diffusive motion, which is the case here, equation 9 becomes,

$$
\beta(q, T)=\beta_{1} \frac{\exp [-2 \gamma(q) T]-1+2 \gamma(q) T}{2[\gamma(q) T]^{2}}+\beta_{\infty} .
$$

Essentially, this means that XSVS can be used to measure the same quantity - the ISF - which was measured by XPCS. As mentioned above, since for most X-ray detectors the minimum integration time is significantly smaller than the period between frames in a continuous time series, XSVS can be used to "push" the experiments to faster timescales than achievable by XPCS. The main drawback of XSVS is that the signal-to-noise from single image measurements is obviously lower and increasing it requires many realizations of the same measurements.

\section{Data Analysis Tools}

\section{A. Scikit-beam}

Multidimensional X-ray data collected at different facilities are traditionally processed and analyzed by a set of graphical user interfaces (GUI's) based on analysis and visualization tools, which are generally not open source and often specific to a particular technique. This poses a significant challenge to 

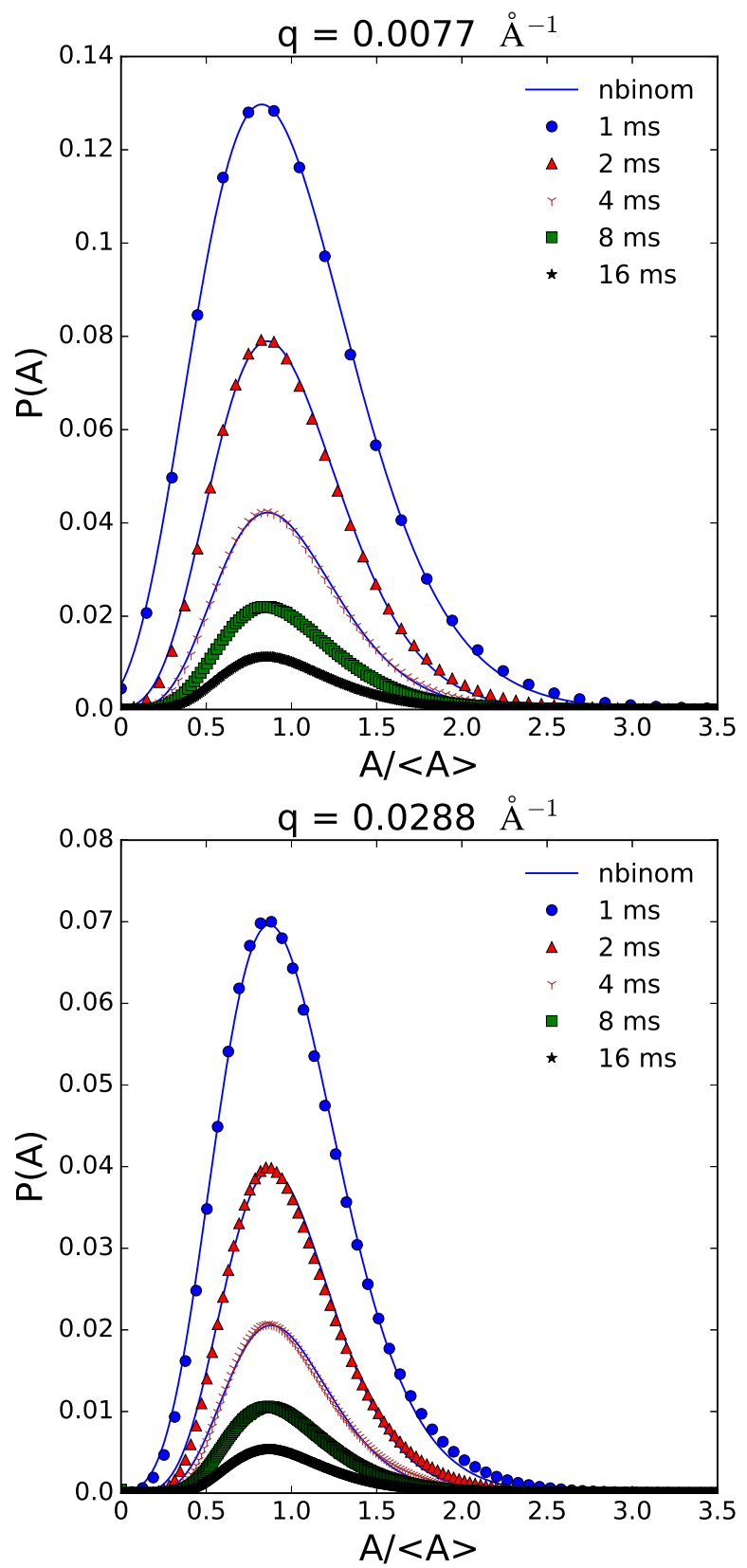

Fig. 5. XSVS analysis for the CoralPor $\mathrm{R}$ sample (Figure 6) shown here from two different $q$ rings, $q=0.77 \times 10^{-2} \AA^{-1}$ and $2.88 \times 10^{-2} \AA^{-1}$ and 5 different integration times $T$ listed in the legend. Solid lines show the fits with the negative binomial distribution function (Equation 7).

the user communities who wish to combine information from different techniques. If they could use the same set of software tools, it would be immensely beneficial to these diverse communities. It is also essential for new user facilities with high data payloads to provide a unified data analysis framework from data generation to the final science publication.

Scikit-beam is a library to provide analysis tools for electron, neutrons and X-ray techniques. The primary objective of Scikit-beam is to develop a comprehensive set of data analysis tools that minimizes technique-specificity and, instead, maximizes code re-usability. Scikit-beam is implemented in the Python programming language, which is open source and already very popular for scientific data analysis. Scikitbeam was initiated at the NSLS-II by the Data Acquisition, Management and Analysis (DAMA) group and has grown to be a multi-facilities concerted effort with contributors from other Department of Energy (DOE) laboratories. A welldefined narrow user interface and a service oriented architecture provide the Scikit-beam with the semi-structured data required to perform complex computations. This service layer is backed with document based databases, allowing NSLSII scientists to store any experimental metadata regardless of the nature of the experiment. The Databroker(Externally facing API for data) and Datamuxer(tools for accessing data in a convenient, workable format) libraries serve as simple points of interaction in Scikit-beam, hiding the complexities of the back-ends and allowing scientists to access data without having to deal with laborious data cleaning/formatting.

The Scikit-beam is not only available for the beamlines of NSLS-II but also to the broader scientific community. Our library supports a wide range of user expertise, from novice to developer. Our intension is to provide $100 \%$ test coverage to all our analysis codes. We are diligent at updating the documentation for the users and developers.

We provide use case Scikit-beam-examples in two formats, Jupyter (a.k.a Ipython notebooks) and python scripts, for the tools included in this package. These examples can be used to learn how to build analysis workflows using the tools included in the Scikit-beam, and more advanced users will be able to incorporate Scikit-beam into their own analysis scripts or programs. More details about using Scikit-beam, and installation instructions can be found in the Scikit-beam documentation page http://scikit-beam.github.io/scikit-beam/.

The XPCS analysis tools are included in Scikit-beam library in the correlation module while the XSVS analysis tools can be found in the speckle module. There are several examples in Scikit-beam-examples using real data collected at NSLS-II beamlines or other facilities and showcase real features impacting analysis procedures such as background subtraction, masking a beamstop shadow on the detector or unwanted pixels, averaging etc. The XPCS and XSVS analysis pipelines that are used here are available under the CHX_Results_Pipelines repository (https://github.com/NSLS-II-CHX/CHX_Results_Pipelines/ Publications/IEEE_August_2016_XPCS_XSVS_anlysis).

\section{B. One-time correlation analysis}

The input parameters for the one-time correlation analysis function in the Scikit-beam package are num_lev, num_bufs, labels, images. The function outputs $g^{(2)}(q, t)$, lag_steps

The "images" variable points to the series of speckle patterns used in the XPCS analysis. The variables "num_lev" and "num_bufs" correspond to the number of levels and number of buffers used to define the logarithmic time-sampling scheme in the calculation of the multi-tau one-time correlation function. Essentially, a scheme with one level and a num_bufs equal to the total number of frames corresponds to a linear 
time sampling, also referred to as a "brute force" correlator, where every image is correlated with every other image before averaging over equal time intervals $t$. Increasing the number of multi-tau levels num_lev and decreasing the number of points in each level (num_bufs) results in a logarithmic time sampling where the signal to noise is increased by averaging together several single images when calculating correlation functions at longer times $t$ [4]. The one-time correlation analysis implemented here uses a symmetric normalization scheme which was shown to reduce the noise level compared to a standard normalization scheme [4]. While with standard normalization, the correlation functions are normalized by $\left\langle I\left(q, t_{0}\right)\right\rangle^{2}$ according to equation 1 , the symmetric normalization calculates,

$$
g^{(2)}(q, t)=\frac{\left\langle I\left(q, t_{0}\right) I\left(q, t_{0}+t\right)\right\rangle}{\left\langle I\left(q, t_{0}\right)\right\rangle\left\langle I\left(q, t+t_{0}\right)\right\rangle} .
$$

The correlation functions are calculated (averaged) over all the "initial" times $t_{0}$ and over coarse-grained regions of nominally equivalent pixels defined through a labeled array of the same shape as the detector images. The Scikit-beam tools allow the definition of customized of ROIs of simple shapes, such as, rings, rectangles, circles, etc. Fig. 6 represents the "labels" marked on the image data. The multi-tau one-time

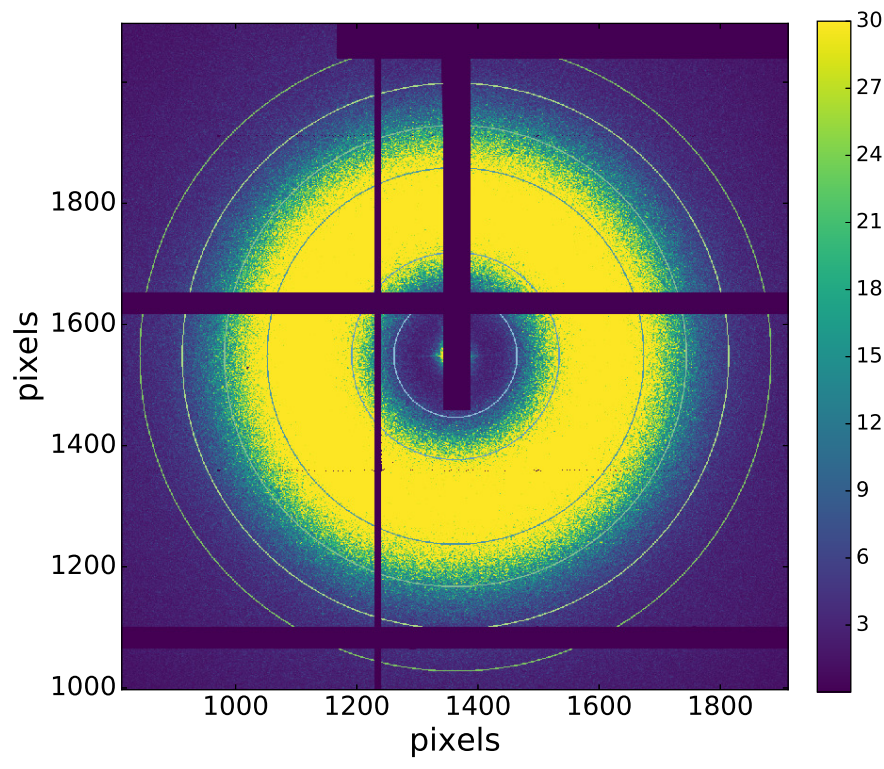

Fig. 6. Ring "labels" (q rings) marked on the CoralPor $\AA$ [8] averaged image (average of 10000 images with $2 \mathrm{~ms}$ integration time). The following $q$ rings are shown; $q=7.70 \times 10^{-3} \AA^{-1}, \quad 1.29 \times 10^{-2} \AA^{-1}, \quad 2.35 \times 10^{-2} \AA^{-1}$, $2.88 \times 10^{-2} \AA^{-1}, 3.41 \times 10^{-2} \AA^{-1}$ and $3.93 \times 10^{-2} \AA^{-1}$ with $\Delta q$ $\simeq 1.0 \times 10^{-4} \AA^{-1}$.

correlation implemented here follows a code which was initially developed by Mark Sutton and collaborators in the Yorick programming language [10].

1) Example for linear time sampling: Here we are presenting an example for a linear time sampling correlator where the number of levels is 1 and the number of buffers is set to
3. For this "brute force" method the number of levels must be one while the number of buffers can be set to any number smaller or equal to the maximum number of available frames and corresponding to the maximum correlation time the user desires to calculate in the correlation functions.

TABLE I

BRUTE FORCE METHOD EXAMPLE. NUMBER OF LEVELS=1 AND NUMBER OF BUFFERS $=3$

\begin{tabular}{||c|c|c|c||}
\hline Brute force level & Effective sampling time & Lag times (buffers) & $\mathrm{I}(\mathrm{t})$ \\
\hline $\mathrm{l}=0$ & $\mathrm{t}_{s}$ & $\mathrm{~b}=0,1,2$ & $\mathrm{I}_{i}$ \\
\hline
\end{tabular}

2) Example for logarithmic (multi-tau) time sampling: Here we present an example in which the number of levels are equal to four, and the number of buffers are equal to 8 . The effective sampling times, lag times and corresponding scattered intensities $I(t)$ for different multi-tau levels are shown in (Table: II). When calculating a multi-tau correlation, no additional averaging is done during the first 8 lag times. Therefore, individual frames are used for the time correlation in the zero level. The effective sampling time is doubled by averaging adjacent frames for longer time lags. In the level one frames 1 and 2 are averaged, 3 and 4 are averaged, and so forth. Except level zero, all other levels perform time correlation for half of the buffers. Therefore, the number of buffers shall always be an even number.

This Scikit-beam one-time correlation function outputs the one-time correlation results, $g^{(2)}(q, t)$ and the corresponding lag_steps, at the times (delays) the correlation is computed.

\section{Two-time correlation analysis}

The two-time correlation analysis module in Scikit-beam uses similar input arguments as the one-time correlation function, num_lev=1, num_bufs, num_imgs, labels, images and outputs three matrices for $C\left(q, t_{1}, t_{2}\right), t_{1}, t_{2}$.

The two-time correlation functions are usually calculated using a linear time sampling. In this case, the number of levels always has to be equal to one, and the number of buffers has to be equal to the number of images used for time $t_{1}$ while "num_imgs" is how many images is used for time $t_{2}$ for the analysis. The "labels" and "images" are the same as described above in the one-time correlation analysis. Usually, the Scikit-beam two-time correlation analysis plots the twotime correlation results, $C\left(q, t_{1}, t_{2}\right)$ as a contour plot in a coordinate system defined by the two times $t_{1}$ and $t_{2}$ (see Fig. 4).

\section{XSVS analysis}

The input variables are images, labels, timebin_num, number_of_img, max_cts. The "labels" and "images" used in the XSVS analysis correspond to the same variables described above in one-time correlation analysis. The "timebin num" is the integration time and the "number_of_img" is the desired number integration steps. The "max_cts" is the brightest pixel in any ROI in any image in the image set.

The Scikit-beam XSVS function outputs the probability of detecting photons "prob_all", and the standard deviation of 
TABLE II

MUlTI-TAU EXAMPLE. NUMBER OF LEVELS $=4$ AND NUMBER OF BUfFERS $=8$

\begin{tabular}{||c|c|c|c||}
\hline Multi-tau level & Effective sampling time & Lag times (buffers) & $\mathrm{I}(\mathrm{t})$ \\
\hline $1=0$ & $\mathrm{t}_{s}$ & $\mathrm{~b}=0,1,2,3,4,5,6,7$ & $\mathrm{I}_{i}$ \\
1 & $2 \mathrm{t}_{s}$ & $8,10,12,14$ & $\frac{1}{2}\left(\mathrm{I}_{i}+\mathrm{I}_{i+1}\right)$ \\
2 & $4 \mathrm{t}_{s}$ & $16,20,24,28$ & $\frac{1}{4}\left(\mathrm{I}_{i}+\mathrm{I}_{i+1}+\mathrm{I}_{i+2}+\mathrm{I}_{i+3}\right)$ \\
3 & $8 \mathrm{t}_{s}$ & $30,38,46,54$ & $\frac{1}{8}\left(\mathrm{I}_{i}+\mathrm{I}_{i+1}+\mathrm{I}_{i+2}+\mathrm{I}_{i+3}+\mathrm{I}_{i+4}+\mathrm{I}_{i+5}+\mathrm{I}_{i+6}+\mathrm{I}_{i+7}\right)$ \\
\hline
\end{tabular}

it, "std_dev".

1) Example for timebin_num and number_of_img: :

When the "time bin number" is 2 and the number of images is 100 , the integration times are 2, 4, 8, 16, 32, and 64. Therefore, the "number_of_img" is the maximum desired integration time can be used for the analysis.

\section{RESUlts AND Discussion}

Using the Scikit-beam data analysis tools, a multi-tau onetime correlation analysis was performed on a data set recorded from the Silica colloidal sample. The recorded time series consisted in $10000 \mathrm{X}$-ray scattering patterns collected each with a $2 \mathrm{~ms}$ integration time. Here we used a logarithmic time sampling with "num_lev"= 12 and "num_bufs"= 8 for the multi-tau auto correlation. The correlation functions, calculated using equation 11, are shown in Fig. 3 (a) for 11 different scattering ( $q$ rings) of this data set.

A two-time correlation analysis was also performed on the same data set as the multi-tau analysis. The two-time correlations used a linear time sampling to obtain $C\left(q, t_{1}, t_{2}\right)$ for $t_{1}$ and $t_{2}$ ranging from $2 \mathrm{~ms}$ (shortest time interval recorded here) to $20 \mathrm{~s}$ (10000 images with $2 \mathrm{~ms}$ counting time per pattern). Fig. 4 represents a contour plot of the two-time correlation calculated for a momentum transfer value $\mathrm{q}=4.80 \times 10^{-3} \AA^{-1}$. For clarity, the two-time correlation in Fig. 4 is plotted over the first 1000 scattering patterns (2 seconds).

The two-time correlation function shows without ambiguity that the silica colloids form an equilibrium fluid and hence the correlation times are age-independent. As a consequence, the two-time correlations can be used to calculate one-time correlations, by simply averaging all the "slices" corresponding to different sample ages. As the time sampling is linear in the two-time correlation it will still be linear in the timeaveraged one-time correlations making this process equivalent to a "brute-force" one-time correlation.

The results obtained from the multi-tau auto correlation analysis $g^{(2)}(q, t)$ (blue stars), are shown in Fig. 7 together with those obtained by time averages over the two-time correlation functions (red circles) for four different $q$ rings. As expected, the two calculations are in excellent agreement.

The one-time correlation functions from both analysis methods described above are fitted with equation 2 (Fig. 8) which are then used together with equation 4 to calculate a diffusion coefficient $D_{0}=2.697 \times 10^{5} \AA^{2} s^{-1}$ characterizing the (random) motion of the Brownian colloidal particles in their solvent. The fits to the correlation functions $g^{(2)}(q, t)$ are also used to calculate the ISFs as,

$$
g^{(1)}(q, t)=\sqrt{\frac{g^{(2)}(q, t)-g_{\infty}}{\beta_{2}}} .
$$

The ISFs are shown in Fig. 3 (b) for the same q-values as the intensity autocorrelation functions.

The Scikit-beam, XSVS analysis tools were used to analyze the data from the CoralPor $\AA$ sample shown in Fig. 6. The first step of the XSVS analysis consist in histogramming all the photon counting events in speckle patterns recorded with an integration time $T$ and over similar pre-defined sets of equivalent pixels ("q-rings"). The histograms are used to calculate the probability density $P(A, q, T)$ which essentially measures the probability of detecting $A$ photons, in a region of the detector at momentum transfer $q$ and with a speckle integration time $T$. The experimental probability density at a given value of $q$ is then fitted with the negative binomial distribution function (Equation 7) for different integration times $T$. The fitting parameters in equation 7 are the average scattering intensity $\langle A\rangle$ and the number of coherent modes $M$.

Fig. 5 show typical examples of the XSVS analysis results for two $q$ rings, $q=0.77 \times 10^{-2} \AA^{-1}$, and $2.67 \times 10^{-2} \AA^{-1}$. The solid lines show the fits with the negative binomial distribution function (Equation 7).

The contrast factor can be calculated from the fitting parameter $M$ using equation 8 for different $q$-values and different integration times $T$. The results of this analysis are shown in Fig. 9.

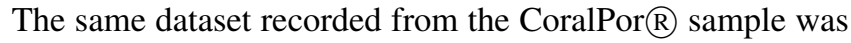
used also to calculate multi-tau one-time correlation functions (Fig. 10) for the same $6 q$ rings (Fig. 6). The different colored markers represent the experimental values for each $\mathrm{q}$ ring. The solid lines show fitted curves using equation 2 . Since the CoralPor $\AA$ sample is static, any correlation time associated with possible experimental instabilities are large-enough to make the correlation functions essentially constant on the timescales probed here and equal to an experimental contrast factor of about $10 \%$.

In conclusion, we have successfully used data analysis tools from the Scikit-beam package to perform XPCS and XSVS analysis at the NSLS-II CHX beamline on two test samples. Our results clearly indicate the reliability and efficiency of the new software tools. These tools are designed for real-time data analysis enabling thus critical experimental decisions on the data acquisition strategy in order to optimize the scientific outcome. 

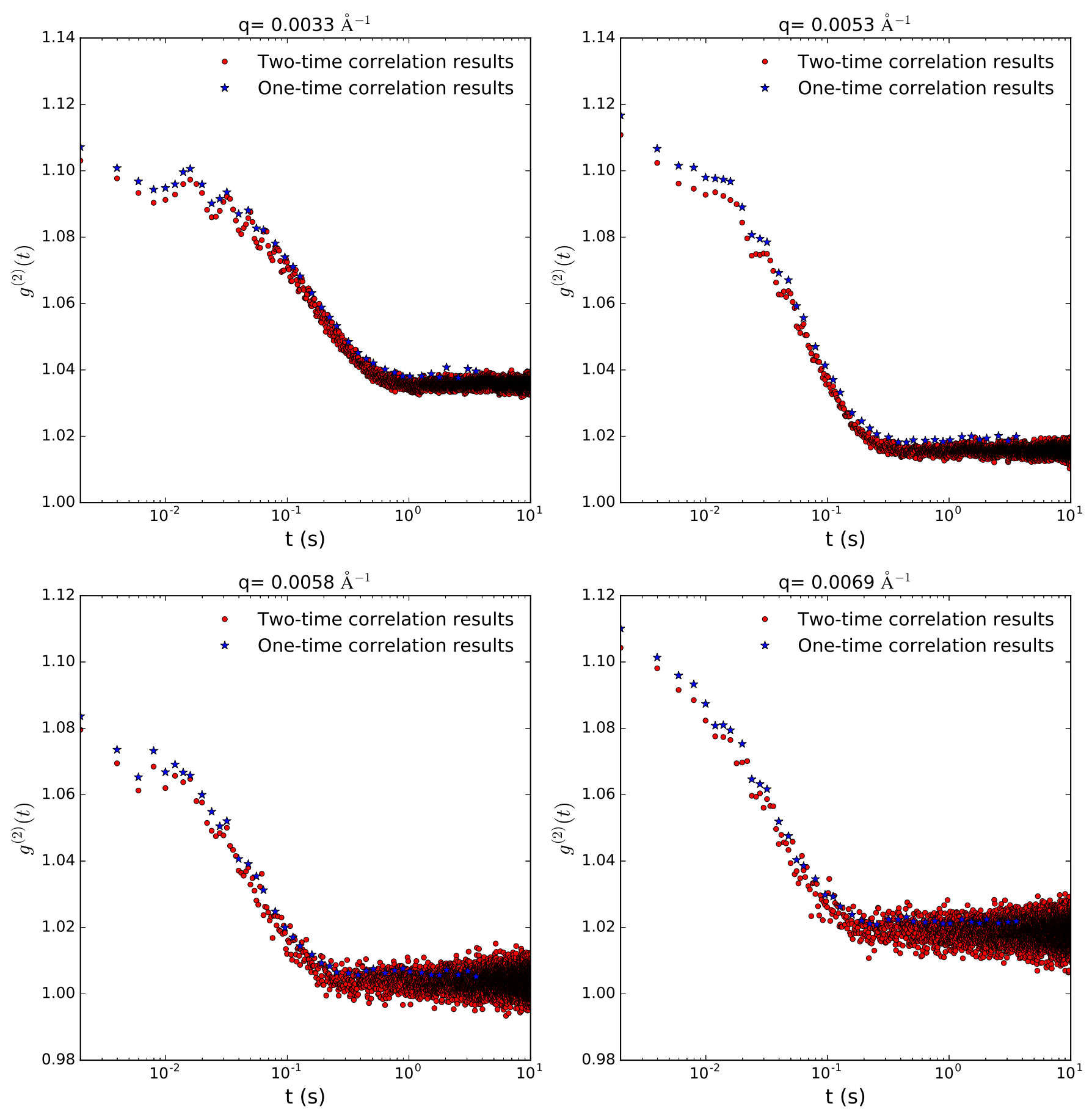

Fig. 7. This figure compares correlation functions calculated for the Silica colloidal suspension for four different $q$ rings. The multi-tau auto correlation functions are shown with the one-time correlation results extracted by averaging two-time correlation functions over time. 


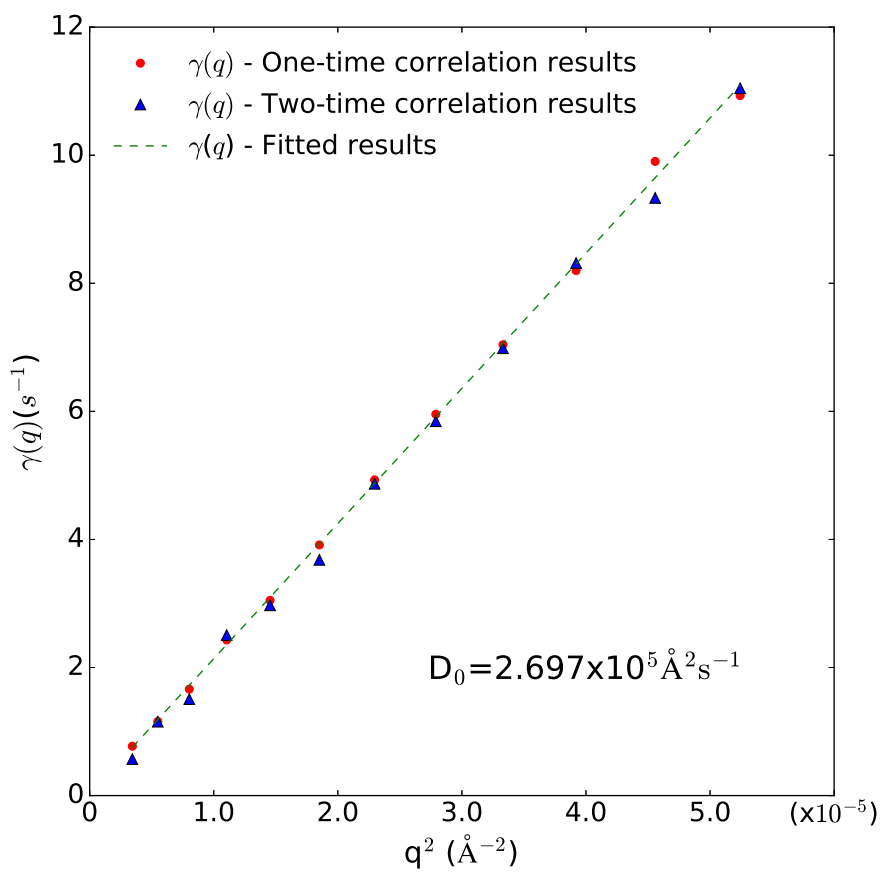

Fig. 8. Relaxation rate $\gamma(q)$ from the colloidal sample obtained by the multi-tau correlation (red circles) and the two-time correlation (blue triangles) analysis plotted against $q^{2}$. The experimental values are fitted by equation 4 (solid line).

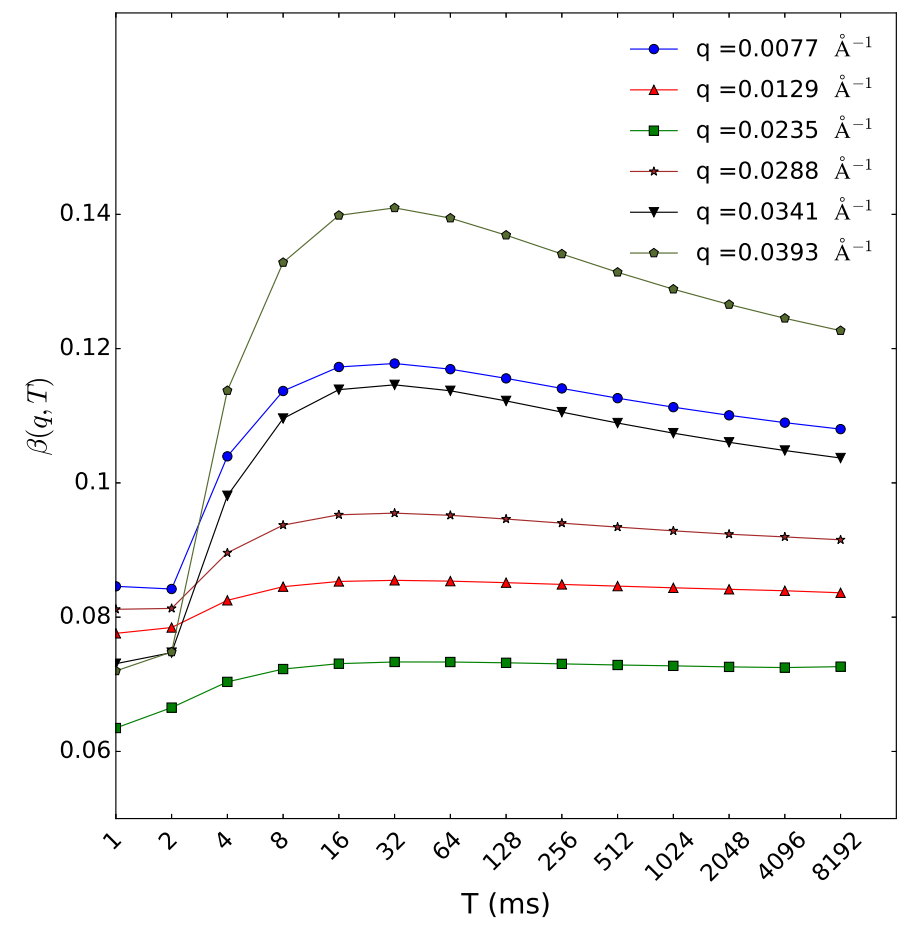

Fig. 9. XSVS anlaysis for CoralPor $\AA \quad$ [8] sample the contrast factor, $\beta$ as a function of integration time

\section{ACKNOWLEDGMENT}

This work at Brookhaven National Laboratory, was supported by the U.S. Department of Energy, Office of Science, Office of Basic Energy Sciences, under Contract No. DE-

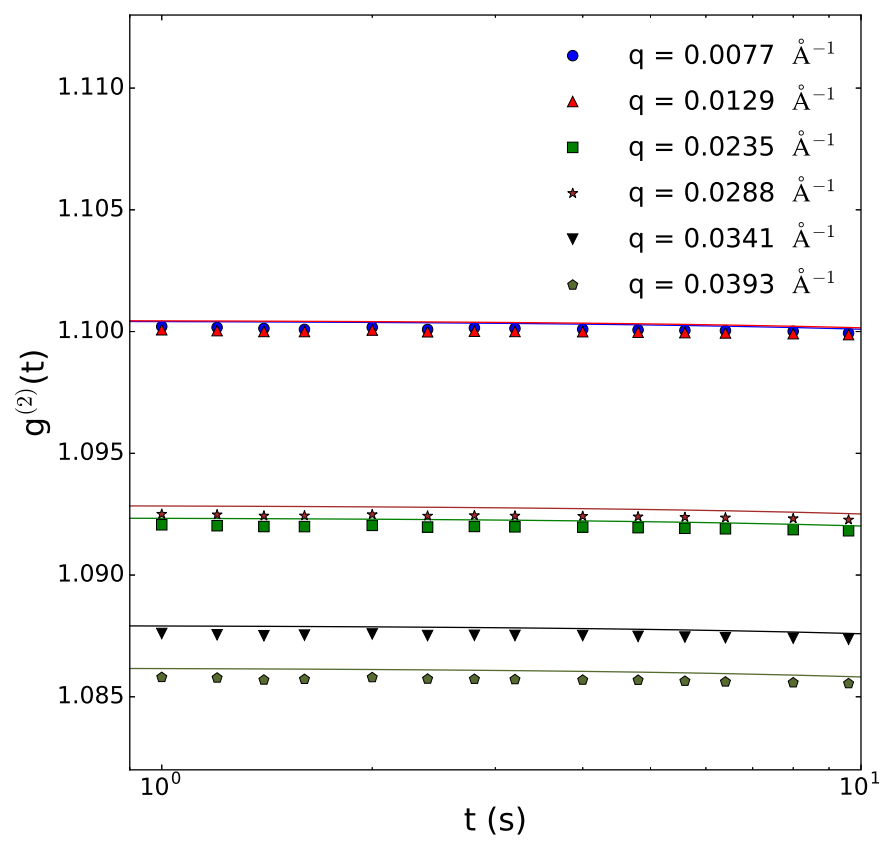

Fig. 10. One-time correlation results obtained for the CoralPor $\AA$ [8] sample for $6 q$ rings. $q=7.70 \times 10^{-3} \AA^{-1}$, $1.29 \times 10^{-2} \AA^{-1}, \quad 2.35 \times 10^{-2} \AA^{-1}, \quad 2.88 \times 10^{-2} \AA^{-1}$, $3.41 \times 10^{-2} \AA^{-1}$ and $3.93 \times 10^{-2} \AA^{-1}$. The different colored markers represent the experimental values for each $q$ rings while, the same color lines represent the fitted values from the equation 5 .

SC0012704, and the Laboratory Directed Research and Development (LDRD) program. The authors would like to thank Yuriy Chushkin from the ESRF for sharing his XPCS code. The authors would like to acknowledge the support from the entire BNL Computer Science Initiative, $\mathrm{Li} \mathrm{Li}$ and Kenneth Lauer at the NSLS-II, DAMA group, and Claudio Mazzoli and Andi Barbour at the Coherent Soft X-ray (CSX-1) beamline at NSLS-II. The authors would also like to acknowledge the support from NSLS-II Scientific staff, NSLS-II controls group and contributors of the Scikit-beam.

\section{REFERENCES}

[1] M. Sutton, A review of X-ray intensity fluctuation spectroscopy, Compt. Rend. Phys., 9, 657-667, 2008.

[2] BNL Photon Sciences about NSLS-II, webpage, https://www.bnl.gov/ps/nsls2/about-NSLS-II.php.

[3] A. Fluerasu, O. Chubar, K. Kaznatcheev, J. Baltser, L. Wiegart, K. Evans-Lutterodt, M. Carlucci-Dayton and L. Berman, Analysis of the optical design of NSLS-II coherent hard X-ray beamline, 2011

[4] D. Lumma, L. B. Lurio, S. G. J. Mochrie and M. Sutton Area detector based phton correlation in the regime of short data batches: Data reduction for dynamic X-ray scattering, Rev. Sci. Instrum., 71, 9, 3274, 2000.

[5] K. Rajan, Informatics for material sceince and Engineering, Elsevier Inc, 2013.

[6] D. W. Huang, B. T. Sherman and R. A. Lampicki, Systematic and integartion analysis of large gene lists using DAVID bioinformatics resources, Nature Protocols, 4(1), 44, 2009.

[7] M. C. Schatz, B. Langmead and S. L. Salzberg, Cloud computing and DNA data race, Nat. Biotechnol., 28(7), 691-693, 2010.

[8] SCHOTT CoralPor Porus Glass, http://www.us.schott.com/english/ download/06.12.13-final-datasheet-coralpor-porous-glass-new.pdf 
[9] M. Sutton, S. G. J. Mochrie, T. Greytak, S. E. Nagler,L. E. Berman, G. A. Held and G. B. Stephenson, Observation of speckle by diffraction with coherent X-rays, Nature, 352, 608, 1991.

[10] M. Sutton, K. Laaziri, F. Live and F. Bley, Using coherence to measure two-time correlation functions, Opt. Express, 11, C22682277, 2003.

[11] B. J. Berne and R. Pecora, Dynamic light scattering with applications to chemistry, biology and physics, Dover, New York, 2000.

[12] L. Li, P. Kwasniewski, D. Orsi, L. Wiegart, L. Cristofolini, C. Caroma and A. Flerasu, Photon statistics and speckle visibility spectroscopy with partially cohernt X-rays., J. Synchrotron Rad., 21, 1288-1295, 2014.

[13] A. Fluerasu, A. Moussaid, A. Madsen and A. Scofield, Slow dynamics and aging coloidal gels studied by $x$-ray photon corrrelation spectroscopy, Phy. Rev. E, 76, 010401, 2007.

[14] C. DeCaro, V. N. Karunaratne, S. Bera, L. B. Lurio, A. R. Sandy, S. Narayanan, M. Sutton, J. Winans, K. Duffin, J. Lehuta, N. Karonis, $X$-ray speckle visibility spectroscopy in the single-photon limit, J. of Synchrotron Rad., 20, 332-338, 2013.

[15] I. Inoue, Y. Shinohara, A. Watanabe, Y. Amemiya, Effect of shot noise on X-ray speckle visibility spectroscopy, Opt. Express 20, 26878-26887, 2012.

[16] S. O. Hruszkewycz, M. Sutton, P. H. Fuoss, B. Adams, S. Rosenkranz, K. F. Ludwig, W. Roseker, D. Fritz, M. Cammarata, D. Zhu, S. Lee, H. Lemke, C. Gutt, A. Robert, G. Grübel, G. B. Stephenson, High Contrast X-ray Speckle from Atomic-Scale Order in Liquids and Glasses, Phys. Rev. Lett., 109, 185502, 2012.

[17] R. Bandyopadhyay, A. S. Gittings, S. S. Suh, P. K. Dixon and D. J. Durian, Speckle-visibility spectroscopy: A tool to study time-varying dynamics, Rev. Sci. Instrum. 76, $093110,2005$.

[18] L. Mandel, Fluctuations of photon beams and their correlations, Proc. Phys. Soc., 72, 1037, 1958

[19] L. Mandel, Fluctuations of photon beams: the distribution of the photoelectrons, Proc. Phys. Soc., 74, 233, 1959. 